\title{
Ethnomedicinal and Ecological studies of some weeds in sugarcane fields of Villupuram district
}

\author{
S. Dhanam \\ PG and Research Department of Botany, Arignar Anna Government Arts College, \\ Villupuram - 605602, Tamil Nadu, India \\ E-mail address: sdhanam2@gmail.com
}

\begin{abstract}
The present communication pertains to ethnobotany of major weeds of sugarcane fields in Villupuram district, Tamil Nadu. Present study was conducted in ten selected sites of Villupuram district in the sugarcane crop fields. Random quadrate method was adopted for studying phytosociological attributes of weeds. A total of 80 plant species belonging to 25 families were identified, out of which 46 plant species are medicinally important to cure different diseases in human beings directly. Amaranthaceae and Euphorbiaceae was the dominant family seen during observation. Frequency, Relative frequency, Density and Relative density of the species were calculated.
\end{abstract}

Keywords: Weeds; Sugarcane field; Ethnobotany

\section{INTRODUCTION}

Plants are to be found everywhere. Man tries to grow only the sort of plants that he wants and the original inhabitants of the soil become useless to him are called as weeds. When land is cultivated to raise crops, weeds spring up naturally along with the crop plants. Quite a number of plants considered as weeds in modern sciences have significant value in ethnobotany. Ethnobotany is a multidisciplinary science defined as the interaction between plants and people. The relationship between plants and human culture is not limited to the use of plants for food, clothing and shelter but also includes their use for religious ceremonies, ornamentation and health care. The World Health Organization (WHO) has estimated that up to $80 \%$ of the world's populations rely on plants for their primary health care (Ramesh, 2008; Akaneme et al., 2008). Weeds are unwanted and undesirable plants growing in a place where some other desirable plants are grown or where no plantation is needed at all. The plants growing in agricultural fields, having more negative values and competing with the main crops for soil, water, nutrients etc are known as weeds (Ali et al., 2003). However, weed is a relative term loaded with value endowed by human beings in relation to their own activities and it is in anthrocentric concept rather than an absolute quality. Wild plants grow in all type weather condition but winter and rainy season is most conductive for the growth of plants. These weeds would be great source of herbal medicines.

Allopathic drugs have brought a revolution throughout the world, but the plant based medicines have its own status (Ahmad 2003). The local uses of plants as a cure are common particularly in those areas, which have little or no assess to modern health services. Hence 
due to less communication means, poverty, ignorance and unavailability of medicinal facilities, most people of especially rural people still forced to practice traditional medicines for their treatment, and also forgetting about indigenous knowledge of plants. But most of the people especially old people still posses the knowledge about wild resourses (Zhang 1996). Nath et al. (2007) described ethnomedicinal aspects of 38 species of weeds of Darrang district of Assam. According to Saika and Hassain (2005) weeds are highly efficacious as medicine against common diseases and other health problems of man. Hence an attempt has been made to survey the ethnomedicinal weeds present in the sugarcane fields of Villupuram district, Tamil Nadu.

\section{MATERIALS AND METHODS}

\section{1. Study area}

The Villupuram district extends over an area of 8204.63 sq. Km, is situated in the south eastern portion of Tamil Nadu. It is bounded on the north by Thiruvannamalai and Kanchipuram districts on the east by the Bay of Bengal, on the south by the district of Cuddalore and on the west by Salem and a part of Dharmapuri districts. The average maximum and minimum temperature ranges from $32.78^{\circ} \mathrm{C}$ to $24.08^{\circ} \mathrm{C}$ respectively. The district lies between $11^{\circ} 57^{\prime} \mathrm{N}$ latitude and $79^{\circ} 32^{\prime} \mathrm{E}$ longitude.

\section{2. Methodology}

Present study was conducted in ten selected sites of Villupuram district in the sugarcane crop fields. Random quadrate method was adopted for studying phytosociological attributes of weeds. Quadrate of 1'x 1' $\mathrm{m}$ were laid down and hence a sum of 60 quadrates was laid. All the weeds from each quadrate were collected separately in polythene bags. The information regarding the local name, plant parts used, name of the diseases cured and the process of administration were collected with the help of rural people, village vaidyas and aged elders. A questionnaire was prepared in the local language for collection of ethic information and interviews were conducted. The collection of information was accompanied by the collection of voucher specimens. The plants were pressed, following the standard technique (Cunningham, 2001). Identification of collected specimens was done with the help of literature. Ethnomedicinal data obtained in the field were complied and compared with the published references of Srivastava et al. (2000), Prajapathi and Khana (2004) and Jadeja et al. (2004). Frequency, Relative frequency, Density and Relative density of the species were calculated by using formulae.

$$
\text { Frequency }(\%)=\frac{\text { Total number of quadrats in which the species occur }}{\text { Total number of quadrats studied }} \times 100
$$

$$
\text { Relative Frequency }(\%)=\frac{\text { Frequency of individuals of a species }}{\text { Total frequency of all species }} \times 100
$$




\section{Density $=\frac{\text { Total number of individuals of a species in all quadrats }}{}$ Total number of quadrats studied}

$$
\text { Relative Density }(\%)=\frac{\text { Density of individuals of a species }}{\text { Total density of all species }} \times 100
$$

\section{RESULTS AND DISCUSSION}

Present study is undertaken with a view to explore the source, purpose and method of use of different plant resources of Villupuram district. The present survey was done to record the overall relationship of the local people with plant resources. The information obtained from the various sources of the area of study has been given in the Table 1.

Table 1. Survey of ethonomedicinal plants in the sugarcane field.

\begin{tabular}{|c|c|c|c|c|c|}
\hline S. No. & $\begin{array}{c}\text { Botanical } \\
\text { Name }\end{array}$ & Family & $\begin{array}{l}\text { Vernacular } \\
\text { Name }\end{array}$ & Parts used & Uses \\
\hline 1. & 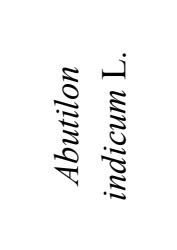 & 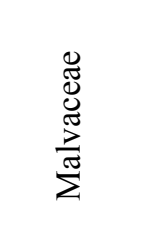 & $\begin{array}{l}\text { 㐏 } \\
\text { 音 }\end{array}$ & 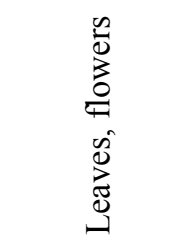 & 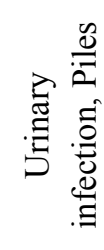 \\
\hline 2. & 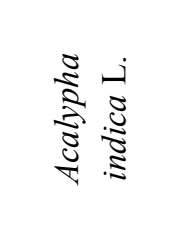 & 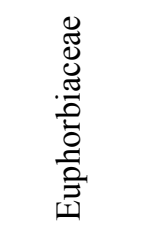 & 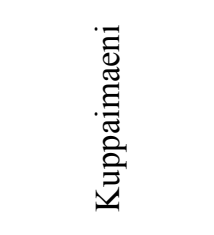 & $\begin{array}{l}\frac{\overrightarrow{0}}{0} \\
\frac{0}{2} \\
\frac{0}{0} \\
\vdots \\
3\end{array}$ & 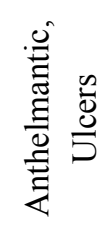 \\
\hline 3. & 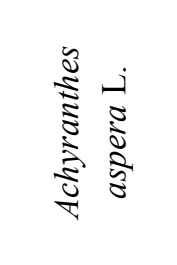 & 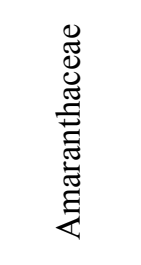 & 忞 & 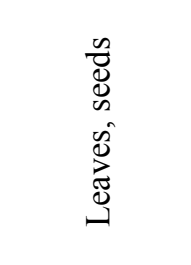 & 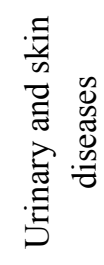 \\
\hline 4. & 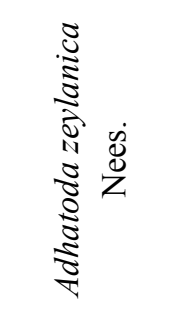 & 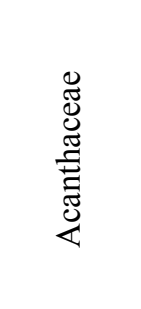 & $\begin{array}{l}\frac{\pi}{0} \\
0 \\
\frac{0}{\pi} \\
\frac{0}{4} \\
\end{array}$ & 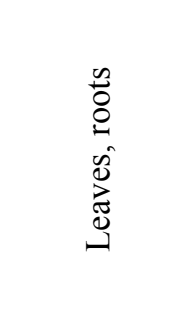 & 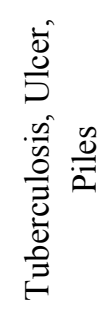 \\
\hline
\end{tabular}




\begin{tabular}{|c|c|c|c|c|c|}
\hline 5. & 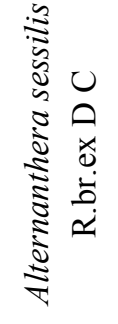 & 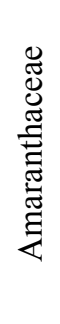 & 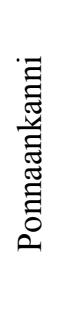 & $\begin{array}{l}\frac{\overrightarrow{0}}{a} \\
\frac{a}{2} \\
\frac{0}{0} \\
\frac{1}{3}\end{array}$ & 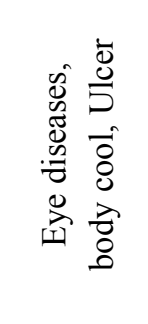 \\
\hline 6. & 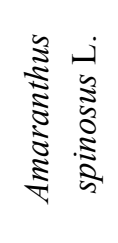 & 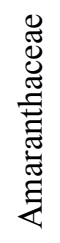 & 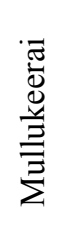 & $\begin{array}{l}\frac{\vec{a}}{0} \\
\frac{0}{2} \\
\frac{0}{0} \\
\frac{1}{3}\end{array}$ & 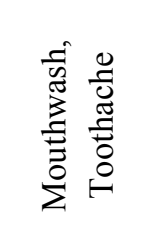 \\
\hline 7. & 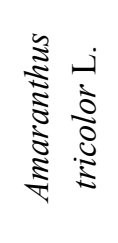 & 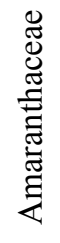 & 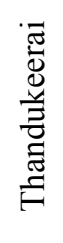 & 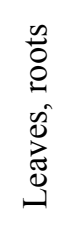 & 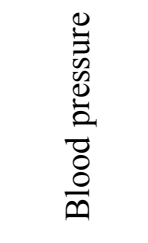 \\
\hline 8. & 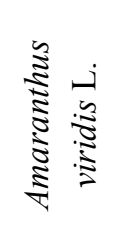 & 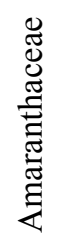 & 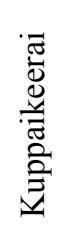 & 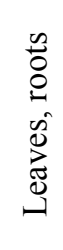 & 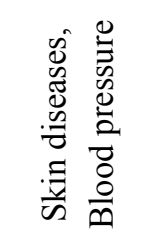 \\
\hline 9. & 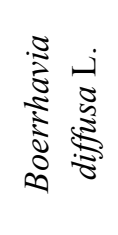 & 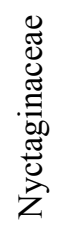 & 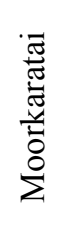 & $\begin{array}{l}\frac{\vec{a}}{0} \\
\frac{a}{2} \\
\frac{0}{0} \\
\frac{1}{3}\end{array}$ & 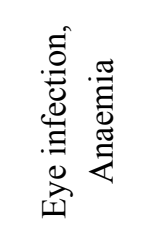 \\
\hline 10. & 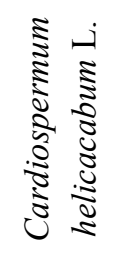 & 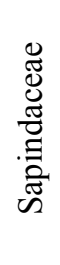 & 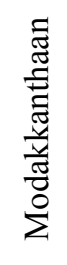 & $\begin{array}{l}\frac{\vec{a}}{a} \\
\frac{a}{2} \\
\frac{0}{0} \\
\frac{1}{3}\end{array}$ & 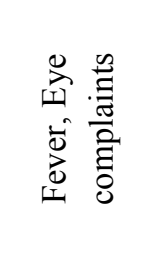 \\
\hline 11. & 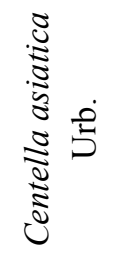 & 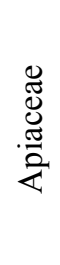 & $\begin{array}{l}\bar{\pi} \\
\frac{\pi}{\pi} \\
\bar{\pi} \\
\end{array}$ & $\begin{array}{l}\frac{\overrightarrow{0}}{0} \\
\frac{\pi}{2} \\
\frac{0}{0} \\
\frac{1}{3}\end{array}$ & 唫. 苞 \\
\hline
\end{tabular}




\begin{tabular}{|c|c|c|c|c|c|}
\hline 12. & 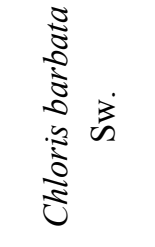 & $\begin{array}{l}\mathscr{J} \\
\mathbb{J} \\
0 \\
0\end{array}$ & 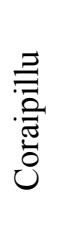 & 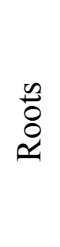 & 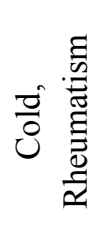 \\
\hline 13. & 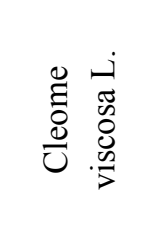 & $\begin{array}{l}\mathscr{\Xi} \\
\tilde{J} \\
\tilde{\Xi} \\
\tilde{\Xi} \\
\tilde{U}\end{array}$ & 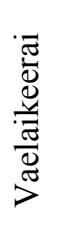 & $\begin{array}{l}\frac{\overrightarrow{0}}{\frac{\pi}{2}} \\
\frac{0}{0} \\
\frac{0}{3} \\
\sum_{3}^{2}\end{array}$ & 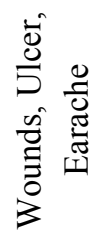 \\
\hline 14. & 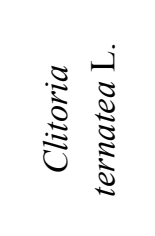 & 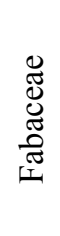 & 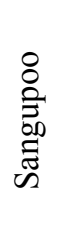 & 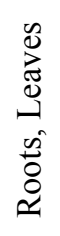 & 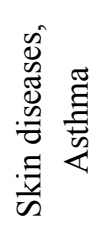 \\
\hline 15. & 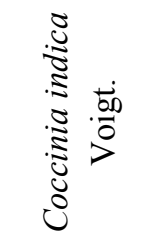 & 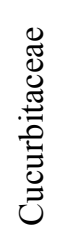 & 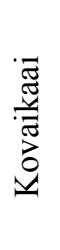 & 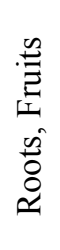 & 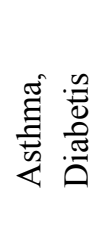 \\
\hline 16. & 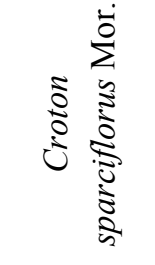 & 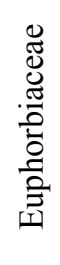 & 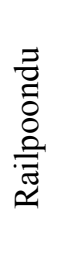 & $\begin{array}{l}0 \\
0 \\
0 \\
2\end{array}$ & 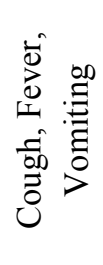 \\
\hline 17. & 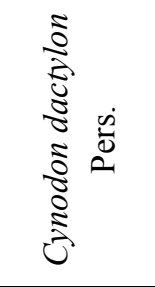 & 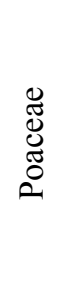 & 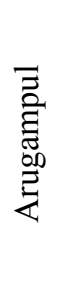 & $\begin{array}{l}\frac{n}{0} \\
\Xi \\
\equiv\end{array}$ & 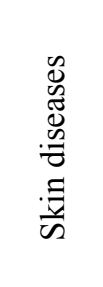 \\
\hline 18. & 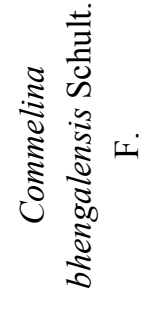 & 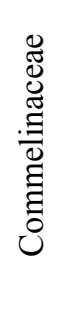 & 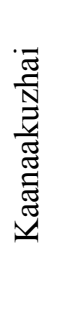 & $\begin{array}{l}\frac{\overrightarrow{0}}{0} \\
\frac{0}{2} \\
\frac{0}{2} \\
\frac{1}{3}\end{array}$ & 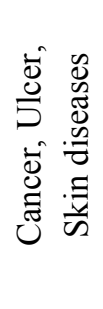 \\
\hline 19. & 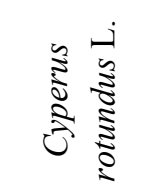 & 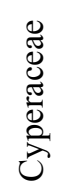 & 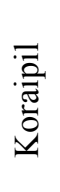 & $\begin{array}{l}\frac{\vec{E}}{\frac{0}{2}} \\
\frac{0}{0} \\
\frac{0}{3} \\
\sum_{3}^{2}\end{array}$ & 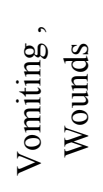 \\
\hline
\end{tabular}




\begin{tabular}{|c|c|c|c|c|c|}
\hline 20. & 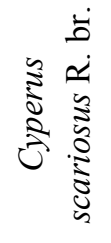 & 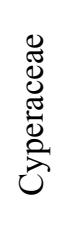 & $\begin{array}{l}\bar{\pi} \\
\overline{0} \\
\frac{0}{0} \\
0 \\
0\end{array}$ & 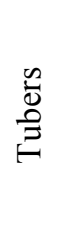 & 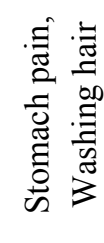 \\
\hline 21. & 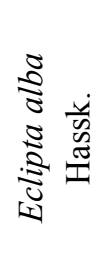 & 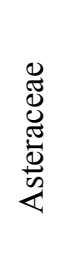 & 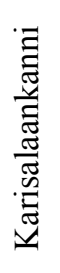 & $\begin{array}{l}\frac{\overrightarrow{0}}{\frac{a}{2}} \\
\frac{0}{0} \\
\frac{0}{3}\end{array}$ & 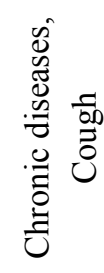 \\
\hline 22. & ن. & 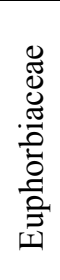 & 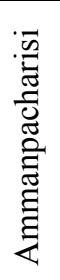 & $\begin{array}{l}\frac{\overrightarrow{0}}{0} \\
\frac{0}{2} \\
\frac{0}{0} \\
\frac{1}{3}\end{array}$ & 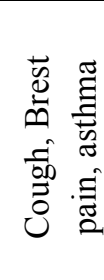 \\
\hline 23. & 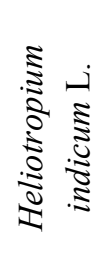 & 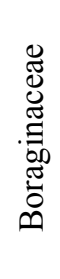 & 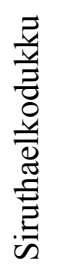 & $\begin{array}{l}\frac{\overrightarrow{0}}{0} \\
\frac{0}{2} \\
\frac{0}{2} \\
\frac{1}{3}\end{array}$ & 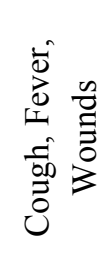 \\
\hline 24. & 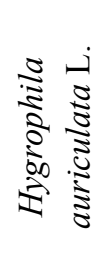 & 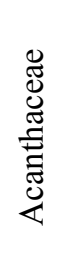 & $\begin{array}{l}\overline{\bar{\Xi}} \\
\bar{\Xi} \\
\ddot{Z}\end{array}$ & 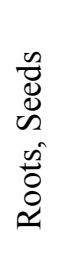 & 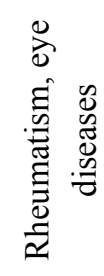 \\
\hline 25. & 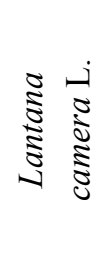 & 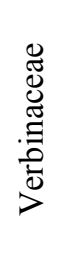 & 竞 & $\begin{array}{l}\frac{\vec{\Xi}}{\frac{0}{2}} \\
\frac{0}{0} \\
\frac{1}{3}\end{array}$ & 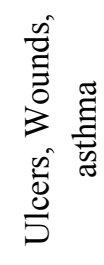 \\
\hline 26. & 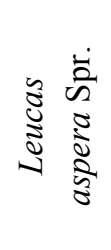 & 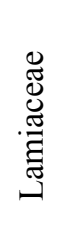 & 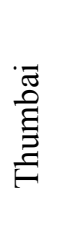 & 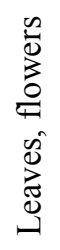 & 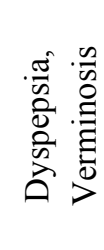 \\
\hline
\end{tabular}




\begin{tabular}{|c|c|c|c|c|c|}
\hline 27. & 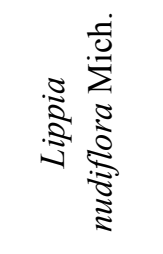 & 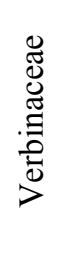 & $\begin{array}{l}\frac{\bar{\pi}}{\pi} \\
\bar{\Xi} \\
\bar{\Xi} \\
0 \\
0\end{array}$ & $\begin{array}{l}\frac{\overrightarrow{0}}{\frac{0}{2}} \\
\frac{0}{0} \\
\frac{0}{0} \\
\sum_{3}^{2}\end{array}$ & 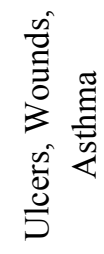 \\
\hline 28. & 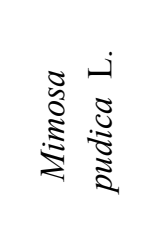 & 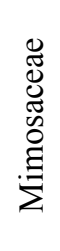 & 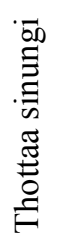 & $\begin{array}{l}\vec{\Xi} \\
\frac{\vec{\sigma}}{2} \\
\frac{0}{0} \\
\frac{1}{3}\end{array}$ & 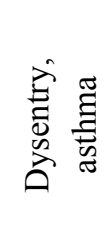 \\
\hline 29. & 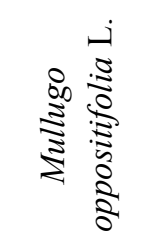 & 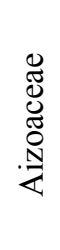 & 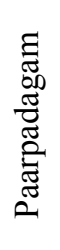 & $\begin{array}{l}\frac{\overrightarrow{0}}{\frac{\pi}{2}} \\
\frac{0}{0} \\
\frac{0}{3} \\
\frac{1}{3}\end{array}$ & 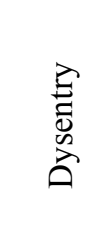 \\
\hline 30 . & 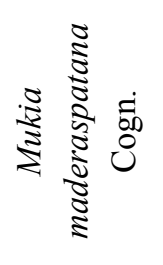 & 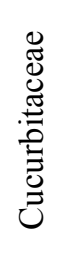 & 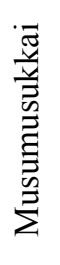 & 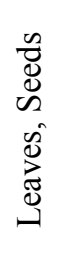 & 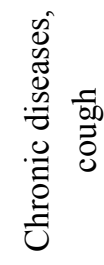 \\
\hline 31. & 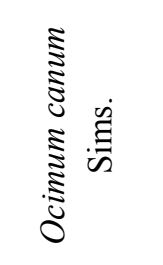 & 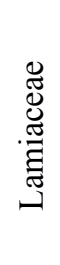 & 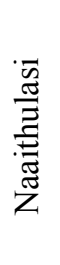 & $\begin{array}{l}\frac{\vec{E}}{0} \\
\frac{0}{2} \\
\frac{0}{0} \\
\frac{1}{3}\end{array}$ & 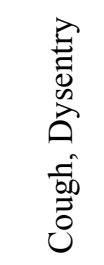 \\
\hline 32. & 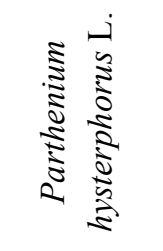 & 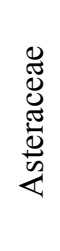 & 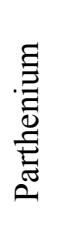 & $\begin{array}{l}\vec{\Xi} \\
\frac{\vec{\sigma}}{2} \\
\frac{0}{0} \\
0 \\
\frac{1}{3}\end{array}$ & $\begin{array}{l}\vec{E} \\
\text { D. } \\
\text { 命 }\end{array}$ \\
\hline 33. & 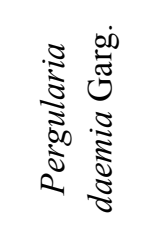 & 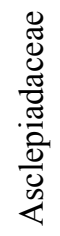 & 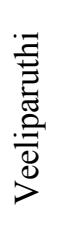 & 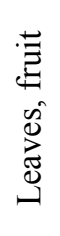 & 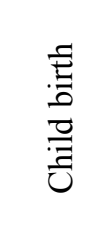 \\
\hline
\end{tabular}




\begin{tabular}{|c|c|c|c|c|c|}
\hline 34. & 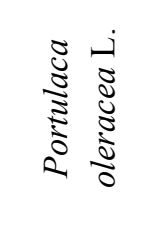 & 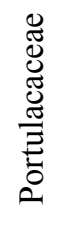 & 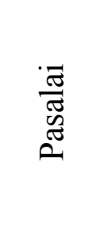 & 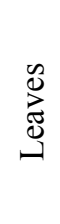 & 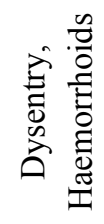 \\
\hline 35. & 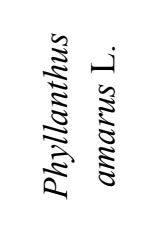 & 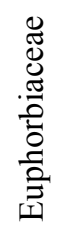 & 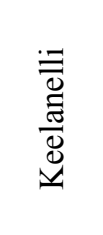 & $\begin{array}{l}\frac{\overrightarrow{0}}{0} \\
\frac{\mathrm{a}}{2} \\
0 \\
0 \\
0 \\
\frac{1}{3}\end{array}$ & 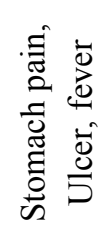 \\
\hline 36. & 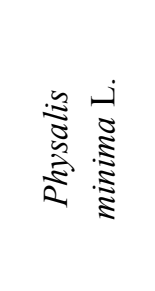 & 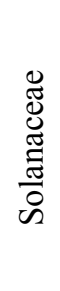 & 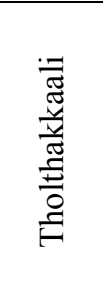 & $\begin{array}{l}\frac{\vec{a}}{0} \\
\frac{0}{2} \\
\frac{0}{0} \\
\frac{1}{3}\end{array}$ & 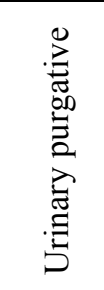 \\
\hline 37. & 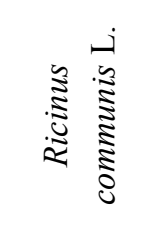 & 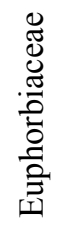 & 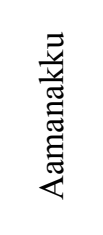 & 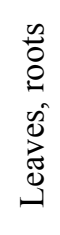 & 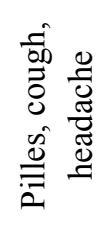 \\
\hline 38. & 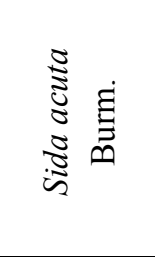 & 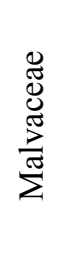 & 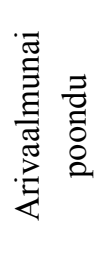 & 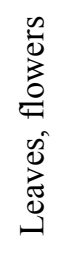 & 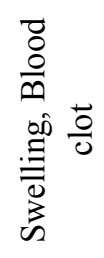 \\
\hline 39. & 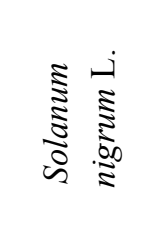 & 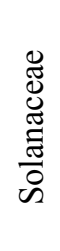 & 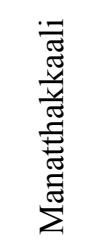 & $\begin{array}{l}0 \\
0 \\
0 \\
0 \\
0 \\
0 \\
0 \\
0 \\
0\end{array}$ & 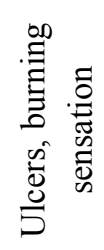 \\
\hline 40. & 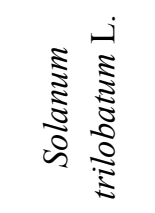 & 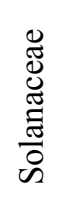 & 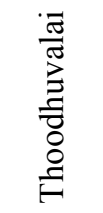 & 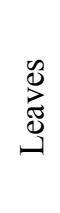 & 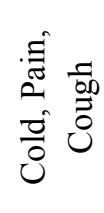 \\
\hline 41. & 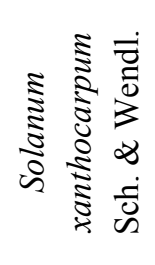 & 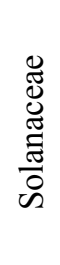 & 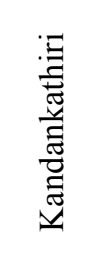 & $\begin{array}{l}\frac{\overrightarrow{0}}{0} \\
\frac{\mathrm{a}}{2} \\
\frac{0}{0} \\
0 \\
3\end{array}$ & 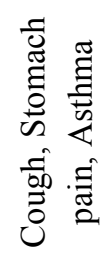 \\
\hline
\end{tabular}




\begin{tabular}{|c|c|c|c|c|c|}
\hline 42. & 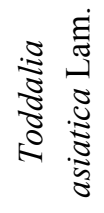 & 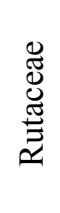 & 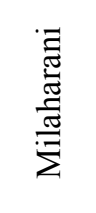 & 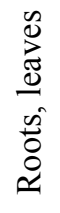 & 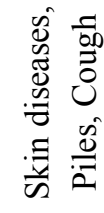 \\
\hline 43. & 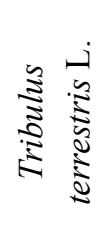 & 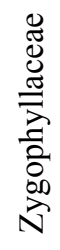 & $\begin{array}{l}: \bar{\Xi} \\
\bar{\Xi} \\
\bar{Z}\end{array}$ & 节 & 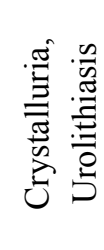 \\
\hline 44. & 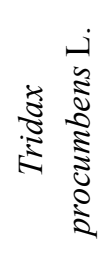 & 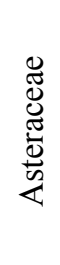 & 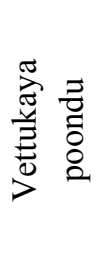 & $\begin{array}{l}\frac{\vec{\sigma}}{a} \\
\frac{0}{2} \\
\frac{0}{0} \\
\frac{1}{3}\end{array}$ & 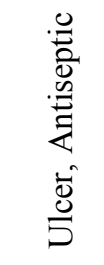 \\
\hline 45. & 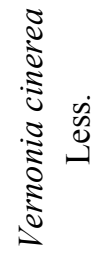 & 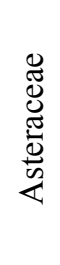 & 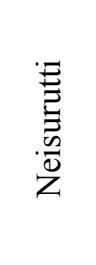 & 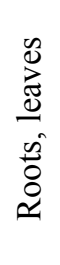 & 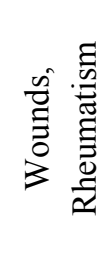 \\
\hline 46. & 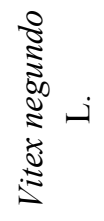 & 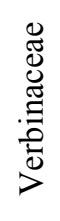 & $\begin{array}{l}\vec{z} \\
0 \\
\text { z }\end{array}$ & $\begin{array}{l}\frac{\vec{J}}{2} \\
\frac{0}{2} \\
\frac{0}{0} \\
\frac{1}{3}\end{array}$ & 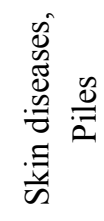 \\
\hline
\end{tabular}

Observation indicated that weed species collected from crop fields are being used to cure different human diseases. Out of 153 weeds studied, 46 weeds are of medicinally important and useful to cure various diseases. Amaranthaceae and Euphorbiaceae was the dominant families present with five generas, Asteraceae (4), Verbinaceae (3), Solanaceae (3), Acanthaceae (2), Cucurbitaceae (2), Cyperaceae (2), Malvaceae (2), Poaceae (2), Lamiaceae (2), followed by Asclepiadaceae, Cleomaceae, Nyctaginaceae, Sapindaceae, Apiaceae, Fabaceae, Commelinaceae, Boraginaceae, Mimosaceae, Aizoaceae, Portulaceae, Rutaceae and Zygophyllaceae. The relative frequency of Cardiospermum, Centella, Chloris and Commelina species was higher than other plants. Phytosociological attributes of sugarcane field is given in Fig. 1, 2, 3 and 4. 

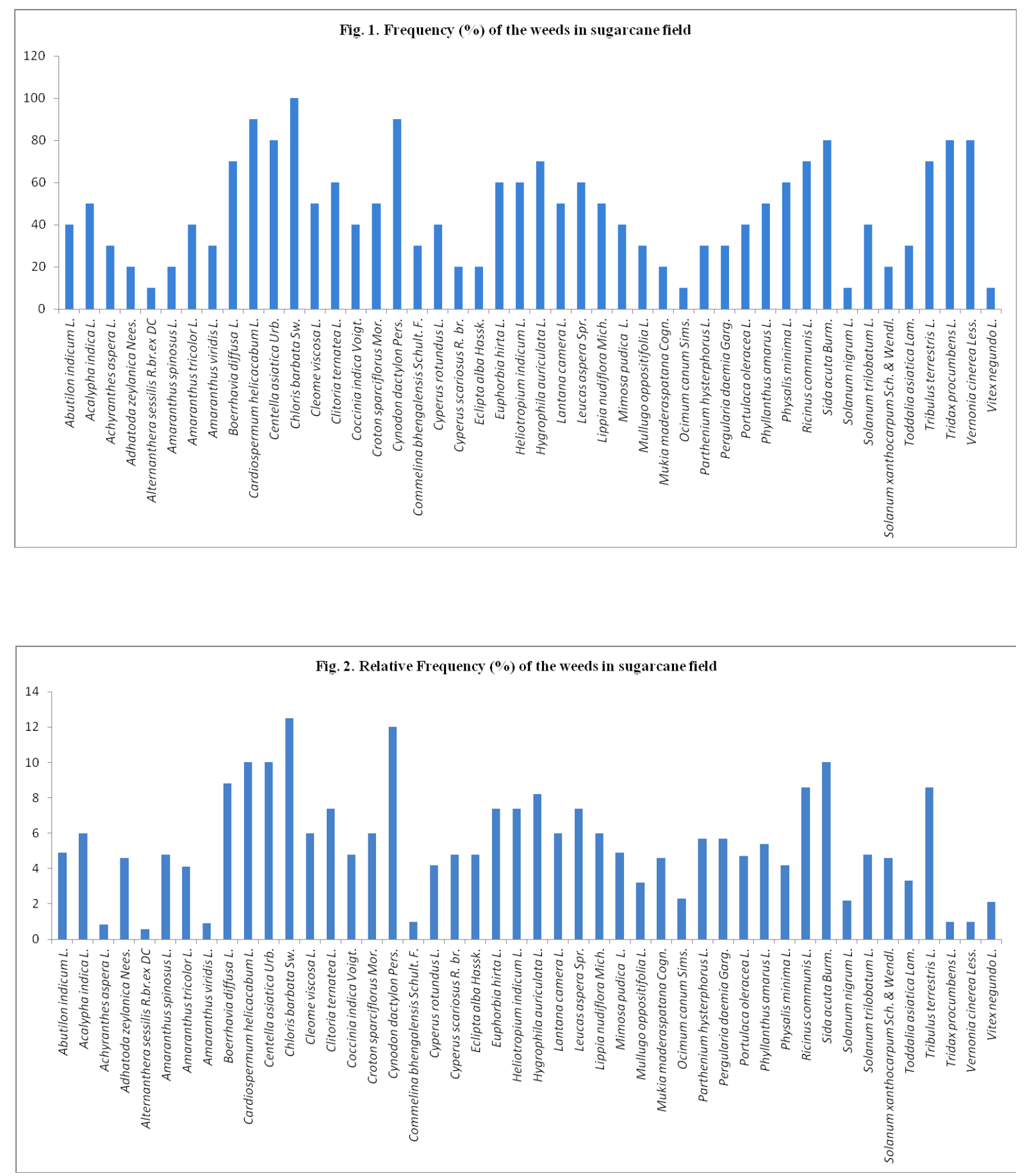

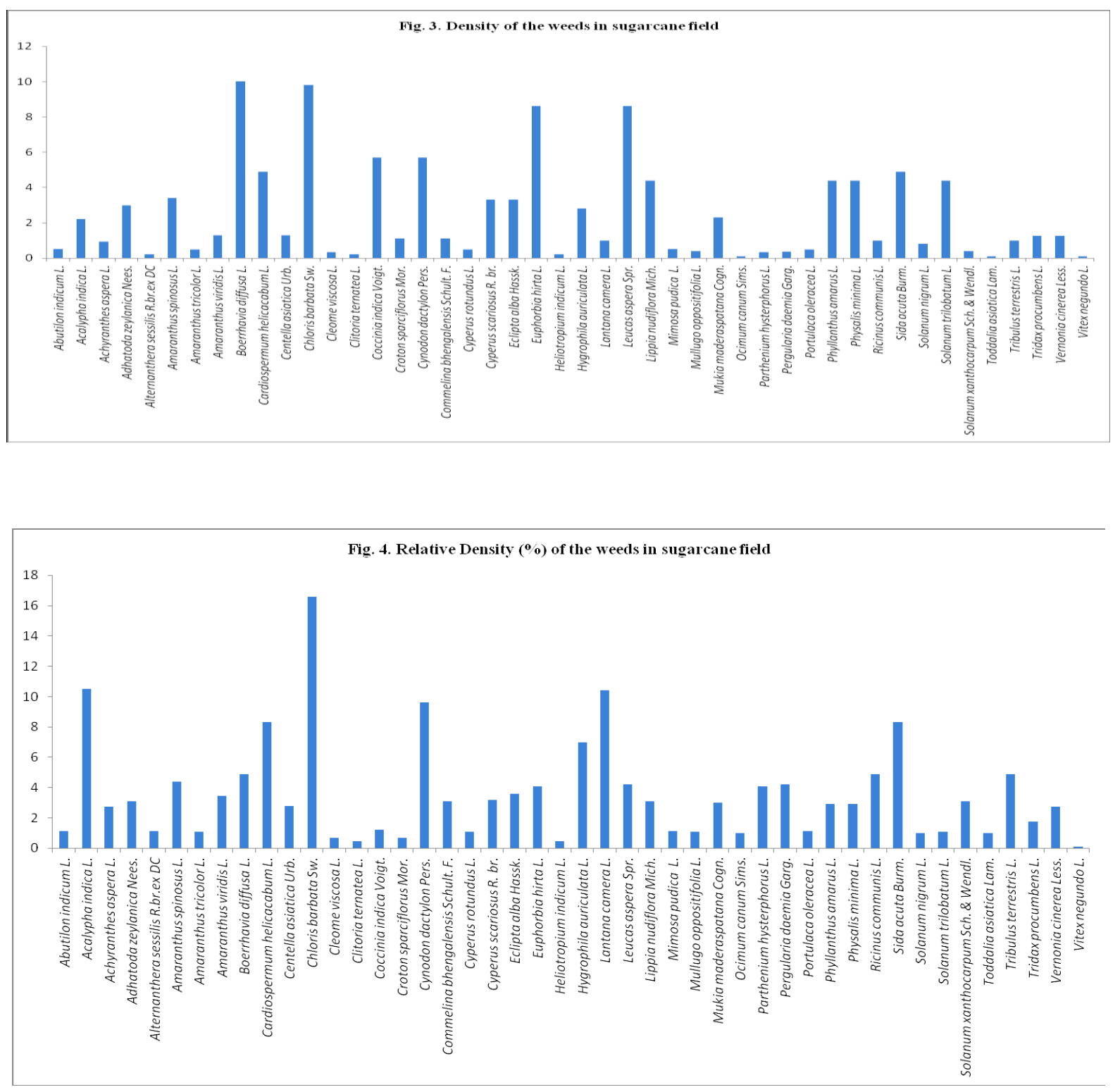

On the traditional uses of weeds a little work has been carried out in India. Weeds play an important role in ayurvedic medicine. Saikia and Hussain (2005) collected information on medicinal aspects of some weeds used by the Ahan and Khamti communities of Sivasagar. The present report coincides with the earlier reports of Adiseshu (1997), Tomar (2009) and Prayagamurthy (2009). Workers like Dangwal et al. (2010) and Perira (1998) have worked on weed flora and their management in other areas of India.

\section{CONCLUSION}

Awareness should be carried out to the local peoples to use these weeds as medicine and to practice them in their day today life. The various uses of these weeds may aid dealers in crude drugs manufactures of plant products or persons interested in the beneficial aspects 
of plants. Hence it is concluded that weeds present in the sugarcane fields can be used as medicines directly or in ayurvedic medicine in large scale.

\section{References}

[1] Adiseshu G. (1997). Ecological aspects of weed flora of sugarcane field at Ankapalle. Ph.D. thesis submitted to the Andhra University, Visakhapatnam.

[2] Ahmed M. (2003). Ethnobotanical and taxonomic studies of economically important plants of Tehsil Attock. M.Phil. thesis, Department of Biological Sciences Quaid- iAzam University, Islamabad.

[3] Akaneme F., African Journal of Biotechnology 7(1) (2008) 6-11.

[4] Ali R., S.K. Khlil, S. M. Raza, H. Khan, Pak. J. Weed Sci. Res. 9(3) (2003) 171-178.

[5] Cunningham A. B. (2001). Applied Ethnobotany people. Wild plant use and conservation, Earthscan Publication Ltd., London, U. K.

[6] Dangwal R. L., A. D. Singh, T. Sharma, A. Sharma, C. Sharma, Pak. J. Weed Sci. Res. 16(1) (2010) 39-45.

[7] Jadeja B. A., D. C. Bhatt, N. K. Udedra, Plant Archives 4(1) (2004) 101-104.

[8] Nath K.K., P. Deda, S.K. Borthakur, Ethnobotany 19(1\&2) (2007) 82-87.

[9] Perirea P., J. Herbs, spices and medicinal plants 6(1) (1998) 43-48.

[10] Prajapathi P.L., P.K. Khane, Plant Archives 4(1) (2004) 113-115.

[11] Prayagamurthy P. (2009). Studies on weeds flora of crop fields of north coastal Andhra Pradesh, Ph.D thesis, Andhra University, Visakhapatnam.

[12] Ramesh R. N., African Journal of Pharmacy and Pharmacology 2(7) (2008) 130-135.

[13] Saikia L.R, I. Hussain, Eco. Env. And Cons. 11(2) (2005) 231-234.

[14] Srivastava A. K., U. Chandra, R. Gopal, S. Srivastava, Ethnobotany (2000) 148-162.

[15] Tomar A., Indian Journal of Traditional knowledge 8(2) (2009) 290-293.

[16] Zhang X., Hamdard Medicus 39(3) (1996) 102.

[17] Suhas J. Vyas, Taslim T. Khatri, Vijay R. Ram, Pragnesh N. Dave, Hitendra S. Joshi, International Letters of Natural Sciences 7 (2014) 16-20. 